\title{
Modelling Dynamic Fronto-Parietal Behaviour During Minimally Invasive Surgery - A Markovian Trip Distribution Approach
}

\author{
Daniel Richard Leff, Felipe Orihuela-Espina, Julian Leong, Ara Darzi, \\ and Guang-Zhong Yang
}

Royal Society/Wolfson Medical Image Computing Laboratory \& Department of Biosurgery and Surgical Technology, Imperial College London, United Kingdom

\{d.leff, f.orihuela-espina, j.leong,a.darzi, g.z.yang\} @imperial.ac.uk

\begin{abstract}
Learning to perform Minimally Invasive Surgery (MIS) requires considerable attention, concentration and spatial ability. Theoretically, this leads to activation in executive control (prefrontal) and visuospatial (parietal) centres of the brain. A novel approach is presented in this paper for analysing the flow of fronto-parietal haemodynamic behaviour and the associated variability between subjects. Serially acquired functional Near Infrared Spectroscopy (fNIRS) data from fourteen laparoscopic novices at different stages of learning is projected into a low-dimensional 'geospace', where sequentially acquired data is mapped to different locations. A trip distribution matrix based on consecutive directed trips between locations in the geospace reveals confluent fronto-parietal haemodynamic changes and a gravity model is applied to populate this matrix. To model global convergence in haemodynamic behaviour, a Markov chain is constructed and by comparing sequential haemodynamic distributions to the Markov's stationary distribution, inter-subject variability in learning an MIS task can be identified.
\end{abstract}

\section{Introduction}

Minimally invasive surgery (MIS) offers considerable benefits to patients in terms of smaller operative incisions, faster convalescence and return to normal activities. However, from the surgeon's perspective, learning to perform MIS is technically demanding. Given the extensive spatial and attentional demands of MIS, marked activation is anticipated in the parietal and prefrontal cortices, since the former is activate during spatially demanding tasks [1] and the latter during novel attentional demands [2]. Hitherto, the cortical contribution to MIS learning has yet to be delineated, possibly due to the challenges of obtaining serial functional measurements during complex procedures.

Portable, discrete, non-invasive functional neuroimaging modalities, such as functional Near Infrared Spectroscopy (fNIRS) are emerging to address these challenges. fNIRS monitors the haemodynamic response to cortical brain activation based upon chromophore related changes in absorption of NIR light ( 700-1000nm) [3]. 
Non-ionising neuroimaging techniques such as fNIRS, offer great potential to monitor characteristics in which brain behaviour adapts to experience and training, a property termed 'neuroplasticity'. Despite this, there has been limited investigation of learning related haemodynamic change using fNIRS [4,5], since analysing dynamic changes in high dimensional data represents a considerable challenge. With this in mind, our aim is to enhance the understanding of MIS learning by monitoring evolving cortical responses with fNIRS. Specifically, our goal is to conduct a global dynamic analysis of multiple cortical subunits (channels) across a fronto-parietal network, since integration of these units likely defines skillful MIS performance [6]. Furthermore, we aim to evaluate intersubject variability in evolving haemodynamic behaviour across learning, as this may characterise differences in innate ability.

The purpose of this paper is to present a novel approach for analysing fNIRS data serially acquired from a group of laparoscopic novices at different time points across learning using spatial interaction modelling techniques. Spatial interaction analysis has been applied to transportation, migration and economic flow, but to our knowledge not to enhance the understanding of fNIRS data sets. High dimensional fNIRS data is first projected into a segregated low dimensional 'geospace', within which sequential transitions between locations can be considered in terms of a series of trips. Construction of a trip distribution matrix enables quantification of inter-regional haemodynamic flow. We hypothesise a flow toward attenuation in haemodynamic behaviour, indicative of learning related neuroplasticity. To model global convergence, a Markov chain was constructed upon the irreducible normalised form of the matrix. Temporal comparisons between individual subject's haemodynamic behaviour and the Markovian stationary distribution reveal variability in learning, possible reflecting aptitude for MIS.

\section{Methods}

\subsection{Subjects and MIS Procedure Set-Up}

Fourteen, healthy right-handed male subjects participated. All subjects were laparoscopic novices (mean age $\pm \mathrm{SD}=20.77 \pm 1.15$ years), with none having performed either supervised or unsupervised laparoscopy. The experimental set-up is illustrated in Fig.1. A Karl Storz laparoscopic stack, Xenon Nova light source, $30^{\circ}$ endoscope, and laparoscopic grasper was used. Subjects were required to perform a laparoscopic task involving repeatedly and accurately locating two points (A and B) on a simulated plastic bowel model with the laparoscopic grasper as shown in Fig.1. A block design experiment was conducted comprising alternating blocks of motor rest (20s) interspersed with the MIS task (20s). Each experimental run consisted of three blocks. Three experimental runs were performed with the camera positioned in the normal view orientation (Classified as N1, N2 and N3 respectively). In between each run, a practice session was conducted involving 3 minutes of task practice. Subjects then performed six further experimental runs, three with the camera rotated $90^{\circ}$ clockwise and three with $90^{\circ}$ counter clockwise rotation. The effect of camera rotation has been previously shown to enhance surgical learning [7]. Finally, the camera was returned to the normal view for a final experimental run (N4). 
(a)

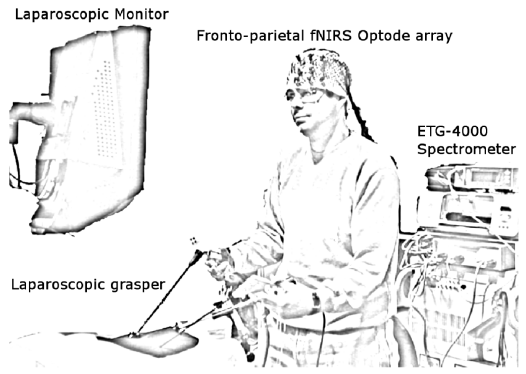

(b)

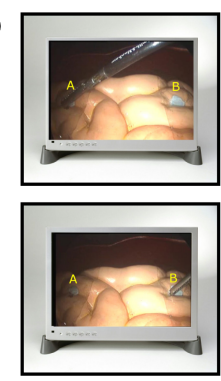

(d)

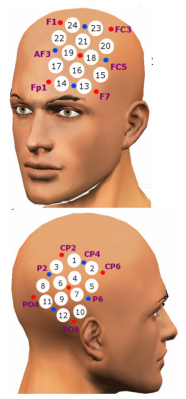

Fig. 1. (a) Experimental setup and videoscopic views of the minimally invasive surgical procedure (b) and (c), corresponding to accurate localisation of the left and right points respectively. Approximate UI 10/10 positions for (d) left PFC and (e) right PC optode arrays.

\subsection{Functional NeuroImaging Acquisition, Preprocessing and Data Embedding}

A 24-channel Optical Topography System (ETG - 4000, Hitachi Medical Co., Japan), comprising two $3 \times 3$ optode probe arrays was used to acquire fNIRS data [8]. Optodes were held within a plastic helmet, affixed to the participant's head with a surgical bandage to secure scalp contact. Optodes were positioned according to the Unambiguously Illustrated 10/10 system [9], such that channels 1-12 were centred on the right parietal cortex (PC) and channels 13-24 on the left prefrontal cortex (PFC).

Optical data was processed using the functional Optical Signal Analysis program (fOSA, University College London, UK [10]). Relative changes in light intensities were converted to changes in haemoglobin data applying the modified Beer-Lambert law. Data was downsampled from $10 \mathrm{~Hz}$ to $1 \mathrm{~Hz}$ to remove physiological noise and detrended to correct for baseline fluctuations. Data was averaged across experimental blocks to increase the signal to noise ratio. Integrity checks were performed to eliminate unreliable data. Finally, a 40D feature space was constructed using 20 task samples from oxygenated haemoglobin $\left(\mathrm{HbO}_{2}\right)$ and deoxygenated haemoglobin $(\mathrm{HHb})$, with each channel containing its own vector. Data was projected into a lowdimensional space applying Isomap [11]. The relationship between embedded locations and averaged regional haemoglobin change are illustrated in Fig. 2.

\subsection{Analysis of Spatial Interactions}

A logarithmic radial grid was imposed to segregate the projected space into a series of sub-regions or cells. These cells group data based upon the similarity of the haemodynamic responses. Spatial interaction analysis seeks to determine spatio-temporal flows and can be used whenever there is a flow of freight, goods or information between an origin and a destination. Here, a given flow describes the transition between two sequential experimental conditions, i.e., $\mathrm{N} 1$ to $\mathrm{N} 2, \mathrm{~N} 2$ to $\mathrm{N} 3$, etc and can be comprehended as a series of trips between cells in the low dimensional space, now considered to be a geospace. 
(a)

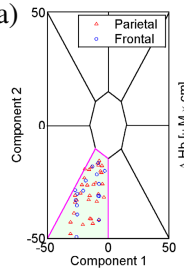

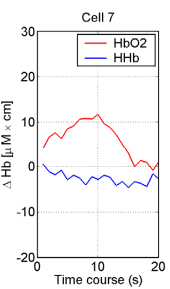
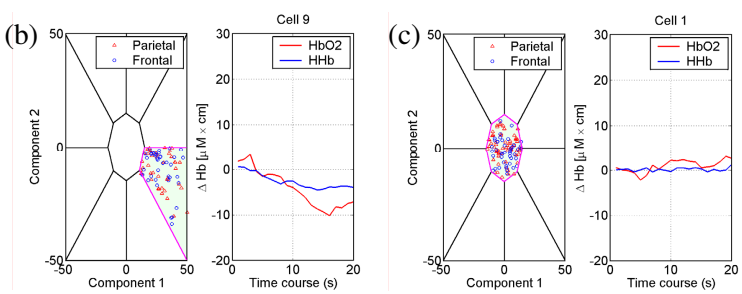

Fig. 2. Example illustrations of the embedded space, partitioned to aid visualisation. Accompanying plots of averaged $\mathrm{HbO}_{2}$ and $\mathrm{HHb}$ change for highlighted quadrants of interest. Plots reflect regions of (a) greatest activity (increase in $\mathrm{HbO}_{2}$ and decrease in $\mathrm{HHb}$ ), (b) attenuated $\mathrm{HbO}_{2}$ responses and (c) minimal signal amplitude at the geospace centre.

\subsubsection{The Trip Distribution Matrix and Gravity Model}

Migration of the embedded fNIRS response in the geospace (called g-Flow) can be captured in an Origin/Destination (OD) matrix, where rows are represented by the locations of origin and columns by locations of destinations. A trip distribution matrix was constructed as follows. The g-flow $F_{i j}$ accounts for all the trips originating from cell $i$ arriving at cell $j$. The trip distribution matrix or transportation matrix $F=\left\{F_{i j}\right\}$ represents the entire flow. In general, the resulting matrix $F$ is sparse in the periphery since most flows are confined to a densely populated region of the geospace.

To ensure irreducibility of the trip distribution matrix $F$, a gravity model defined as below was used:

$$
F_{i j}=G \cdot M_{i}^{\beta_{1}} \cdot M_{j}^{\beta_{2}} \cdot f\left(D_{i j}\right)
$$

where $G$ is a gravitational constant, $M_{i}$ and $M_{j}$ are the volumes or weights of cells $i$ and $j$ respectively. They are calculated as the number of points located in cells $i$ and $j$, and $f\left(D_{i j}\right)$ represents the cost function defined as:

$$
f\left(D_{i j}\right)=\frac{1}{D_{i j}^{\beta_{3}}}
$$

where $D_{i j}$ is the cost of the trip from cell $i$ to $j$ calculated as the Euclidean distance between the geometrical centres of the cells. Applying logarithms to Eqn. (1) and including an error term $\varepsilon$ results in:

$$
\ln F_{i j}=\beta_{0}+\beta_{1} \ln M_{i}+\beta_{2} \ln M_{j}-\beta_{3} \ln D_{i j}+\varepsilon
$$

such that $\beta_{0}=\ln (G)$. This gives a system of equations which can be solved by least squares regression with $\beta_{0,1,2,3}$ being the regression parameters of the gravity model. The normalized updated matrix $\widehat{F}$ provides the input to a Markov chain.

\subsubsection{Markov Prediction of Temporal Flow}

In this paper, a Markov chain [12] is used to model the temporal flow across regions of the geospace to reveal the long-term confluence of haemodynamic behaviour. Knowing the location of a point in the geospace $l(n)$ after $n$ experimental conditions, it is 
possible to forecast the most likely new location $l(n+m)$ after $m$ experimental conditions. The next state in the Markov chain is calculated by the forward equation:

$$
l(n+1)=l(n) P
$$

Hence a Markov chain is fully characterised by a transition matrix $P$ between states, or in this case cells. $\hat{F}$ is by definition a stochastic or probabilistic matrix, that can be fed into the Markov chain, i.e., $P=\hat{F}$. The steady state vector $\pi$ can be interpreted as the likelihood of specific haemodynamic brain responses following extended training on the MIS task.

\subsubsection{Learning Curve Analysis}

For each condition (N1 to N4), the distribution of projected points for all channels of a given subject represent the subject's state vector sv(subject,condition). It is possible to compare $s v$ (subject,condition) against the steady state vector $\pi$, where the difference between these state vectors, can be calculated as the L2 norm $\| s v($ subject, condition $)-\pi \|_{2}$. For each subject, learning curves can be depicted across conditions to evaluate temporal change in haemodynamic behaviour.

\section{Results}

The principal modes of the $g$-flow for all the subjects studied are illustrated in Fig.3. Despite the complexity, flow is toward the centre of the projected geospace, suggesting

(a)

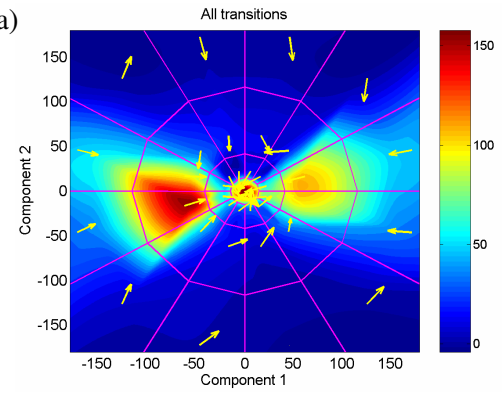

(c)

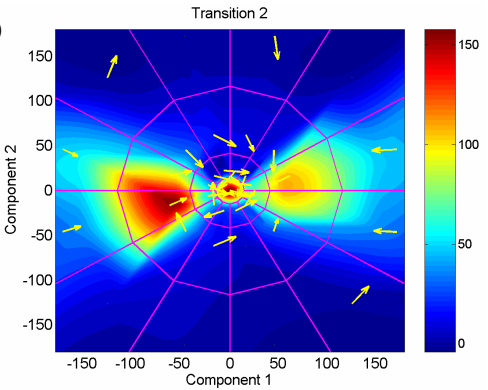

(b)

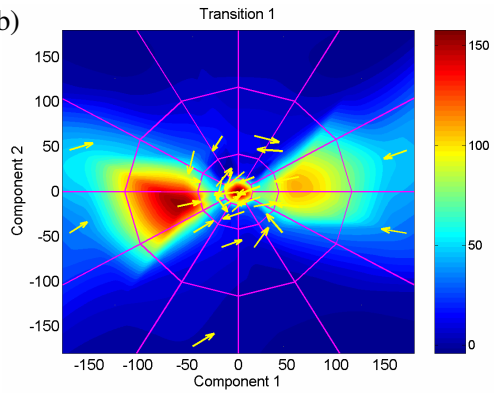

(d)

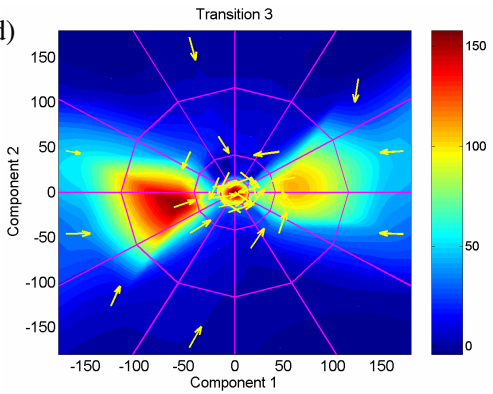

Fig. 3. (a) Principal mode of the flow across the entire experiment and for each transition (b) $\mathrm{N} 1 \rightarrow \mathrm{N} 2$, (c) N2 $\rightarrow \mathrm{N} 3$, and (d) N3 $\rightarrow \mathrm{N} 4$, respectively. Background thermal colour intensity plots represent total volume for $M_{i}$. 
global convergence in cortical haemodynamic behaviour. Interestingly, the greatest convergence in $g$-flow is apparent following the third transition (i.e., N3 to N4), possible indicating learning related haemodynamic change following both extended training and camera rotation.

Dynamic g-flow across each experimental condition is illustrated in Fig. 4, for all the 24 NIR channels used. Despite global convergence, evolution at a local level varies considerably. Nevertheless, certain flow similarities are evident. As reflected in the principal modes of the flow, channels projecting to the lower left of the geospace are likely to deviate in an anticlockwise direction about the origin. This can be appreciated as progressive attenuation in first $\mathrm{HbO}_{2}$ and second in the $\mathrm{HHb}$ response (see Fig.2). Additionally, anticipated within-region flow similarities are apparent (e.g. PC channels 5, 7 and 10; PFC channels 17 and 19). More interestingly, visualising dynamic g-flow unveils inter-regional (fronto-parietal) flow similarity (e.g. channels 5 and 22).

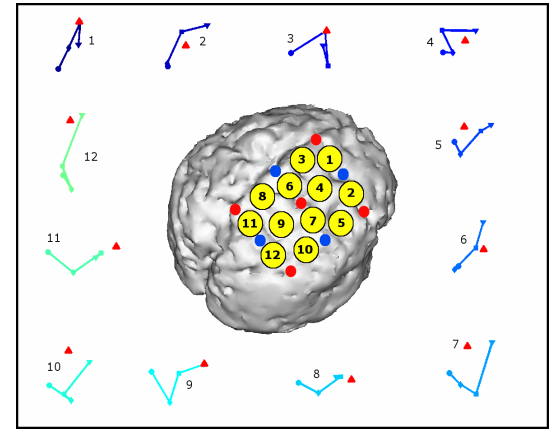

(a)

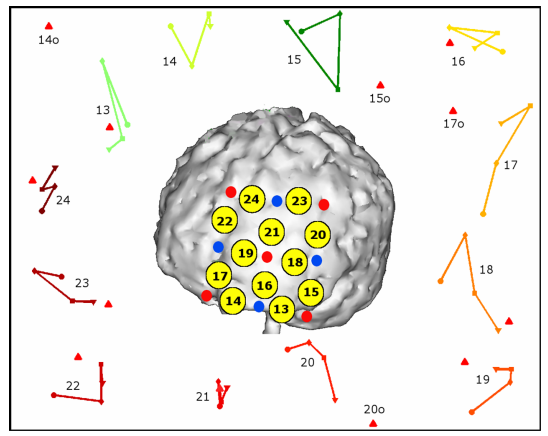

(b)

Fig. 4. Approximate channel locations (yellow circles) for the right PC (a) and left PFC (b), overlaid onto T1 weighted MRI images. Subplots illustrate haemodynamic flow for each labelled NIR channel with respect to the centremost cell (red triangle) in the geospace. Where a particular flow is peripheral, the centremost cell has been highlighted as (o). Location for a given condition is calculated as the k-means centroid of all subjects. Greater flow similarity is observed in the PC versus the PFC.

Table 1. Summary of the Trip Distribution Matrix

\begin{tabular}{c|ccc}
\hline $\begin{array}{c}\text { Geospace } \\
\text { location }\end{array}$ & $\begin{array}{c}\text { Incoming } \\
\text { Flow }\end{array}$ & $\begin{array}{c}\text { Outgoing } \\
\text { Flow }\end{array}$ & $\begin{array}{c}\text { Internal } \\
\text { Flow }\end{array}$ \\
\hline A & 50.0 & 66.0 & 23 \\
B & 14.3 & 34.0 & 0.7 \\
C & 10.0 & 12.0 & 0.7 \\
D & 21.0 & 19.3 & 1.0 \\
E & 22.6 & 22.6 & 1.7 \\
F & 15.0 & 23.0 & 4.7 \\
G & 20.0 & 21.7 & 4.0 \\
H & 30.0 & 20.0 & 11.3 \\
I & 18.3 & 19.7 & 4.0 \\
\hline
\end{tabular}

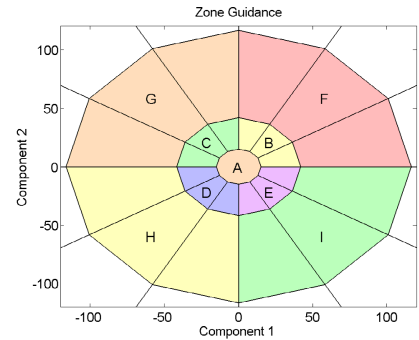


The trip distribution matrix summarised in Table 1 supports the global flow toward attenuated haemoglobin signals. Inter-subject variability in evolving fronto-parietal cortical haemodynamics during MIS learning is illustrated in Fig.5 as a series of learning curves across temporal conditions. Broadly, three different learning curves are observed, although the general trend is one of slow progress toward the steady state.

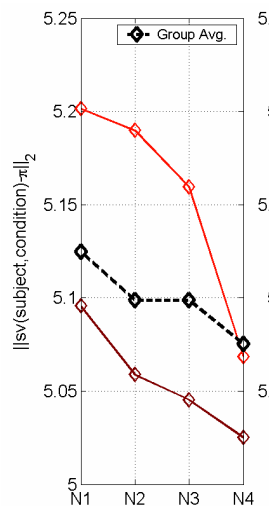

(a)

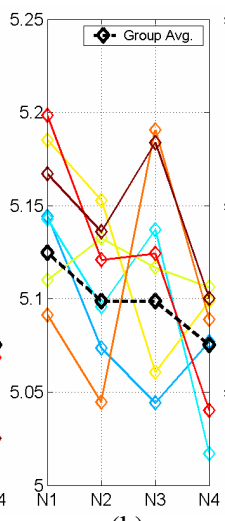

(b)

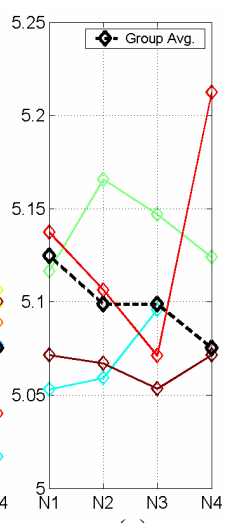

(c)

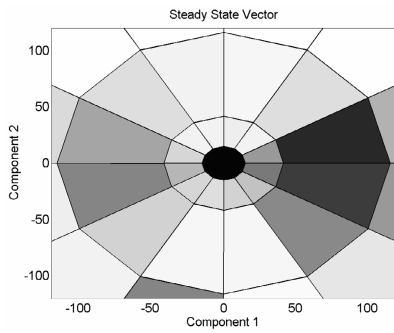

(d)

Fig. 5. Individual subject learning curves, comparing fronto-parietal haemodynamic distributions at each time interval to the stationary distribution. Plots are classified by those subjects who (a) progress towards the steady state at each transition, (b) are closer to steady state at the end of practice but not necessarily at each transition and (c) are further from steady state at the end of training. (d) Empirically determined stationary distribution where intensity of shading represents the probability of each cell acting as the final geospace location. The darkest represents the most likely destination cell and the lightest the least likely.

\section{Discussion and Conclusion}

In this paper, we have proposed a novel approach to analysing longitudinal fNIRS haemodynamic behaviour. Using this framework, it has been possible to observe learning related changes in fronto-parietal haemodynamic flow. Regardless of both anatomical location and/or origin in the geospace, the trip distribution matrix and steady state vector of the Markov chain suggest global convergence toward a region of minimal haemoglobin change. It is hypothesised that this reflects learning related neuroplasticity or more efficient neuronal behaviour following training. It should be noted, however, there may be alternative explanations for global convergence (e.g. habituation). Nevertheless, Markovian modelling permits a probabilistic depiction of the final pattern of cortical haemodynamics, against which a subject's progress through learning can be compared. 


\section{References}

1. Harris, I.M., Egan, G.F., Sonkkila, C., Tochon-Danguy, H.J., Paxinos, G., Watson, J.: Selective right parietal lobe activation during mental rotation: a parametric PET study. Brain 123, 65-73 (2000)

2. Kelly, A.M., Garavan, H.: Human functional neuroimaging of brain changes associated with practice. Cerebral Cortex 15, 1089-1102 (2005)

3. Jobsis, F.: Noninvasive, infrared monitoring of cerebral and myocardial oxygen sufficiency and circulatory parameters. Science 198, 1264-1267 (1977)

4. Hatakenaka, M., Miyai, I., Mihara, M., Sakoda, S., Kubota, K.: Frontal regions involved in learning of motor skill-A functional NIRS study. Neuroimage 34, 109-116 (2007)

5. Leff, D.R., Orihuela-Espina, F., Atallah, L., Darzi, A., Yang, G.Z.: Functional near infrared spectroscopy in expert and novice surgeons - a manifold embedding approach. In: Ayache, N., Ourselin, S., Maeder, A. (eds.) MICCAI 2007, Part II. LNCS, vol. 4792, pp. 270-277. Springer, Heidelberg (2007)

6. Leff, D.R., Elwell, C.E., Orihuela-Espina, F., Atallah, L., Delpy, D.T., Darzi, A., Yang, G.Z.: Changes in prefrontal cortical behaviour depend upon familiarity on a bimanual coordination task: An fNIRS study. Neuroimage 39, 805-813 (2008)

7. Jordan, J.A., Gallagher, A.G., McGuigan, J., McClure, N.: Randomly alternating image presentation during laparoscopic training leads to faster automation to the "fulcrum effect". Endoscopy 32, 317-321 (2000)

8. Sato, H., Kiguchi, M., Kawaguchi, F., Maki, A.: Practicality of wavelength selection to improve signal-to-noise ratio in near-infrared spectroscopy. Neuroimage 21, 1554-1562 (2004)

9. Jurcak, V., Tsuzuki, D., Dan, I.: 10/20, 10/10 and 10/5 system revisited: Their validity as a relative head-surface-based positioning systems. Neuroimage 34, 1600-1611 (2007)

10. Koh, P.H., Glaser, D.E., Flandin, G., Kiebel, S., Butterworth, B., Maki, A., Delpy, D.T., Elwell, C.E.: Functional optical signal analysis: a software tool for near infrared spectroscopy data processing incorporating statistical parametric mapping. J. Biomed. Opt. 12, 64010 (2007)

11. Tenenbaum, J.B., de Silva, V., Langford, J.C.: A global geometric framework for nonlinear dimensionality reduction. Science 290, 2319-2323 (2000)

12. DeGroot, M.H., Schervish, M.J.: Probability and Statistics. Addison-Wesley, Reading (2001) 